\title{
Numerical Study of Thermal-Diffusion and Diffusion- Thermo Effects on Mixed Convective Flow and Mass Transfer in the Presence of MHD over an Accelerating Surface
}

\author{
Girinath Reddy Mottupalle 1,*(D), Dinesh Pobbathy Ashwathnarayana ${ }^{1}$, Basavaraj M Shankarappa ${ }^{1}$, Uma \\ Munivenkatappa ${ }^{1}$
}

1 Department of Mathematics, M. S. Ramaiah Institute of Technology (Affiliated to VTU), Bangalore -560054, Karnataka, India.; mgrmathes@gmail.com (M.G.R.); dineshdpa@msrit.edu (D.P.A.); basavarajms149@gmail.com (M.S.B.); umashivaram@gmail.com (U. M.)

* Correspondence: mgrmathes@gmail.com;

Scopus Author ID 57215905684

Received: 12.11.2020; Revised: 7.12.2020; Accepted: 9.12.2020; Published: 12.12.2020

Abstract: This study investigates the impact of MHD, variable viscosity, thermal-diffusion, and diffusion-thermo effects with the heat source on mixed convection of heat and mass transfer over an accelerating surface. The physical problem's governing equations involve a coupled non-linear partial differential equations, which are transformed to a coupled non-linear ordinary differential equations using a suitable similarity transformation. Numerical computation using shooting technique is adopted to study the physical characteristics of velocity, temperature, and concentration for various values of non-dimensional parameters like thermal diffusion parameter, diffusion-thermo parameter, viscosity parameter, Prandtl number, and strength of the magnetic field are involved in the problem. The obtained numerical results are found to be a good agreement with the earlier works.

Keywords: thermal-diffusion; diffusion-thermo; accelerating surface; variable viscosity; MHD.

\section{Nomenclature:}

Magnetic number $\quad: M=\sqrt{\frac{\sigma_{m} B_{0}{ }^{2} L}{\rho U_{0}}}$

Prandtl number

$$
: \operatorname{Pr}=\frac{\rho v_{\infty} C_{p}}{k}
$$

Eckert number

$$
: E=\frac{b^{2} x^{2}}{C_{p}\left(T_{w}-T_{\infty}\right)}
$$

Schmidt number

$$
: S c=\frac{v_{\infty}}{D_{m}}
$$

Dufour number

$$
: D f=\frac{D_{m} K_{T}\left(C_{w}-C_{\infty}\right)}{C_{s} C_{p} v_{\infty}\left(T_{w}-T_{\infty}\right)}
$$

Soret number

$$
: S r=\frac{D_{m} K_{T}\left(T_{w}-T_{\infty}\right)}{v_{\infty} T_{m}\left(C_{w}-C_{\infty}\right)}
$$

Reynolds number $: R e=\frac{u_{0} x}{v}$

$\eta \quad$ : Dimensionless similarity variable

$r \quad:$ Temperature parameter

$s \quad$ : Heat flux parameter

$g$ : Gravitational acceleration

$f \quad$ : Non dimensionless stream function
Local temperature Grashof number : $G r_{t}=\frac{g \beta\left(T_{w}-T_{\infty}\right) x^{3}}{v_{\infty}{ }^{2}}$

Local mass Grashof number $\quad: G r_{m}=\frac{g \beta^{*}\left(C_{w}-C_{\infty}\right) x^{3}}{v_{\infty}^{2}}$

Viscosity in PSTC case

$$
: \theta_{r}=\frac{T_{r}-T_{\infty}}{T_{w}-T_{\infty}}=-\frac{1}{\gamma\left(T_{w}-T_{\infty}\right)}
$$

Viscosity in PWHCF case $\quad: g_{r}=\frac{T_{r}-T_{\infty}}{T_{w}-T_{\infty}}=-\frac{1}{\gamma\left(T_{w}-T_{\infty}\right)}$

Mass buoyancy parameter $\quad: G c=\frac{G r_{m}}{\operatorname{Re}^{2}}$

Non-dimensional longitudinal coordinate $\quad: \delta=\frac{B x_{0}}{\rho c_{p}}$

Temperature buoyancy parameter $\quad: s=\frac{G r_{t}}{\operatorname{Re}^{2}}$

Constant injection

$$
: V_{w}=m(b v)^{\frac{1}{2}}
$$

$\theta(\eta)$ : Dimensionless temperature in PSTC case

$g(\eta)$ : Dimensionless temperature in PWHCF case

$H(\eta)$ : Dimensionless concentration in PSTC case 
$h(\eta) \quad$ : Dimensionless concentration in PWHCF case

(C) 2020 by the authors. This article is an open-access article distributed under the terms and conditions of the Creative Commons Attribution (CC BY) license (https://creativecommons.org/licenses/by/4.0/).

\section{Introduction}

In the literature survey, several authors come across fluid properties that are not affected by the physical parameters like position, time, temperature, and concentration. Many researchers have mathematically investigated most of the applications in various fields like nuclear waste materials, chemical catalytic reactors, wet-bulb thermometers, geothermal system, food processing, and polymer solution. The researchers have also considered the constant fluid properties without accelerating surface in many industrial and engineering applications. The fluid flow over an accelerating surface is important in many practical applications such as extrusion of plastic sheets, metal spinning, paper production, glass blowing, drawing plastic films, and spinning of fibers, continuous casting of metals, thermal insulation, drying of solids, enhanced oil recovery, and many others. In our day to day life, the combined heat and mass transfer phenomena are observed in the crop damage due to freezing, distribution of temperature and moisture over agricultural fields, formation and dispersion of fog, groves of fruit trees, and environmental pollution.

Lai and Kulacki [1] examined the convective heat transfer flow under the influence of variable fluid viscosity about a porous vertical flat surface in the presence of a saturated porous medium. Mixed double-diffusive convective flows with MHD have a broad range of applications in the area of aeronautical plasma flows, planetary and stellar magnetospheres, electronics, and chemical engineering. The coupled heat and mass transfer by free convection on a steady MHD convective flow through a saturated porous medium with a heat source, subjected to constant heat flux, and Acharya et al. [2] analyzed constant suction velocity over a vertical infinite flat surface. Further, Seddeek [3] examined an unsteady MHD convective flow by natural convection past an upward direction of a semi-infinite plate with a transverse magnetic field along with effects of variable viscosity and radiation. Later, Das et al. [4] studied the natural convective magneto-nanofluid flow and radiative heat transfer past a moving vertical plate.

In theoretical and experimental studies, it has been observed that the concentration gradients play a role in creating an energy flux along with temperature gradients; these activities can be observed in petroleum, chemical engineering processes, isotope separation, and many more applications. In nature, the presence of pure air (real gases) or pure water (real fluids) is impossible, i.e., the air or water is mixed with some foreign concentration or mass. Eckert and Drake [5] first explained the effects of Dufour (thermal-diffusion) and Soret (diffusion-thermo), i.e., the study between the mixture of gases with light molecular weight and medium molecular weight. Later, Kafoussias and Williams [6] studied the effects of Soret and Dufour on simultaneous heat and mass transfer convective flow in the presence of temperature-dependent fluid viscosity. Further, Seddeek [7] investigated the effects of temperature-dependent fluid viscosity on mixed double-diffusive convective flow with suction and blowing over an accelerating flat surface in the presence of Soret and Dufour effects.

Gnaneswara Reddy et al. [8] presented Soret and Dufour's effects on both a steady and unsteady free convective MHD flow with viscous dissipation past a semi-infinite accelerating vertical flat plate through an embedded porous medium by finite element analysis. Later, Girinath Reddy et al. [9-10] studied the effects of variable viscosity and porosity of fluid. Soret 
and Dufour mixed double-diffusive convective flow over an accelerating surface with internal heat generation. Ullah Khan et al. [11] analyzed Soret and Dufour's effects on the unsteady flow of electrically conducting Eyring-Powell fluid over an oscillatory stretching sheet by using convective boundary conditions. Further, Soret and Dufour's effects on thermophoresis MHD radiative flow and heat transfer over a non-linear stretching sheet under chemical reaction have been investigated by Shalini Jain et al. [12]. Soret and Dufour effects on MHD heat and mass transfer of Walter's-B viscoelastic fluid over a semi-infinite vertical plate are considered by Idowu et al. [13]. The combined effects of non-Darcy and magnetohydrodynamic (MHD) forces on free convection of non-Newtonian fluids over a vertical permeable plate through porous medium with thermal radiation, Soret and Dufour effects have been studied by Huang [14].

Suresh Babu et al. [15] investigated the effects of the double double-diffusive mixed convective flow of an incompressible viscous fluid numerically through a vertical heated plate embedded in a non-Darcy porous medium under the influence of variable fluid properties. Irfan et al. [16] explained the features of nanoparticles mass flux conditions and non-uniform heat sink/source on magnetite Oldroyd-B nanofluid. Additionally, heat convective and thermal radiation mechanisms are considered. Jawad Ahmed et al. [17] explored the heat and mass transfer mechanisms by incorporating the features of energy and mass fluxes caused by mass concentration and temperature gradients. Further, space and temperature-dependent heat source/sink influence are considered on fluid thermal characteristics. Further, heat and mass transfer for a Forchheimer model of electrically conducting fluid with Soret and Dufour effects over a vertical heated plate is studied by Nalinakshi et al. [18]. Sravan Kumar et al. [19] gave a theoretical analysis to draw out the flow characteristics of free convective nanofluid flow along with an exponentially accelerating vertical plate in the presence of a magnetic field.

Veera Krishna et al. [20] made elaborate scrutiny on the Soret and Joule effects of MHD mixed convective flow of an incompressible and electrically conducting viscous fluid past an infinite vertical porous plate taking Hall effects into account. Shankar Goud et al. [21] focused on unsteady natural convection MHD flow with heat and mass transfer past an inclined plate. Sohaib Abdal et al. [22] have investigated the multislip effects on the magnetohydrodynamic (MHD) mixed convection unsteady flow of micropolar nanofluids over a stretching/shrinking sheet along with radiation in the presence of a heat source. Tijani Yusuf et al. [23] studied the time-varying hydromagnetic chemically reacting free convective flow of a viscous, incompressible radiating fluid past an infinite oscillating vertical plate with variable surface conditions subject to transverse magnetic field in the presence of temperature-dependent heat source. Soret and Dufour effects are taken into consideration. Sreedhar et al. [24] examined the two-dimensional, unsteady, laminar, magnetohydrodynamic free convective flow through a porous medium past a vertical plate in the presence of heat absorption and chemical reaction is considered. The Dufour effect is taken into account, and the free stream velocity is supposed to follow the exponentially increasing small perturbation law. Anantha Kumar Kempannagari et al. [25] examined the convective heat transfer characteristics on magnetohydrodynamic stagnation point flow of micropolar fluid past an exponentially curved surface. The flow is supposed to be laminar and time-independent. Ayegbusi Florence et al. [26] investigated the problem of unsteady magnetohydrodynamic heat plus mass transfer convective flow over a moveable vertical plate with the influence of thermophoresis and thermal radiation.

Dinesh et al. [27] examined the effects of Forchheimer and radiation adsorption on MHD double-diffusive unsteady dusty viscoelastic Couette flow in an irregular channel with 
chemical reaction. Basavaraj et al. [28] investigated a non-linear mixed convective oscillatory flow embedded with a semi-infinite vertical porous plate in the presence of a uniform magnetic field. Uma et al. [29] studied Forchheimer, Soret, and Dufour's combined effects on free-forced convective dusty viscoelastic Couette flow in an irregular channel under a constant magnetic field. Thermo-diffusion (Soret effect) and diffusion-thermo (Dufour effect) effects on doublediffusive natural convection induced in a horizontal Brinkman porous layer with a stress-free upper boundary are investigated by Ismail Filahi et al. [30]. In the present study, the main novelty is to study numerically the effects of Soret and Dufour on MHD mixed convection heat and mass transfer over an accelerating surface with a heat source in the presence of temperature-dependent fluid viscosity subjected to two different conditions PSTC and PWHCF.

\section{Materials and Methods}

\subsection{Mathematical formulation of the problem.}

We consider a steady (i.e., independent of time), two-dimensional along with $x \& y$ coordinate axes and double-diffusive (temperature and concentration/species) mixed (free \& forced) convection for an electrically conducting, incompressible, laminar, viscous fluid flow under the effect of a uniform transverse magnetic field applied in the upward direction to the flat plate. The flow under consideration of the fluid is due to the external force exerted by the vertical plate's accelerating surface. Here, the coordinate $x$-axis runs along with the vertical plate and $y$-axis is perpendicular to the vertical flat plate, and acceleration due to gravity plays in the opposite direction to the $x$-axis. The linear velocity $u=b x$ along $x$-axis which is due to accelerating surface and $v=v_{w}$ is a constant injection velocity along $y$-direction is maintained. The physical configuration and the coordinate system of the fluid flow are shown in Figure 1, and for this physical system of the fluid flow, the following assumptions are made: (i) effects of Soret (diffusion-thermo) and Dufour(thermal-diffusion) are taken into account; (ii) Joule effect and Lorentz's force are considered; (iii) the molecular transport properties like thermal conductivity and diffusivity are considered as constant; (iv) chemical reactions are not taking place between fluid and species; (v) the variable viscosity is inversely proportional to a linear function of temperature, which is in the form of $\frac{1}{\mu}=\frac{1}{\mu_{\infty}}\left[1+\gamma\left(T-T_{\infty}\right)\right]$ or $\frac{1}{\mu}=$ $a\left(T-T_{r}\right),\left(\right.$ See Lai and Kulacki [1]) where a $=\frac{\gamma}{\mu_{\infty}}, T_{r}=T_{\infty}-\frac{1}{\gamma}$.

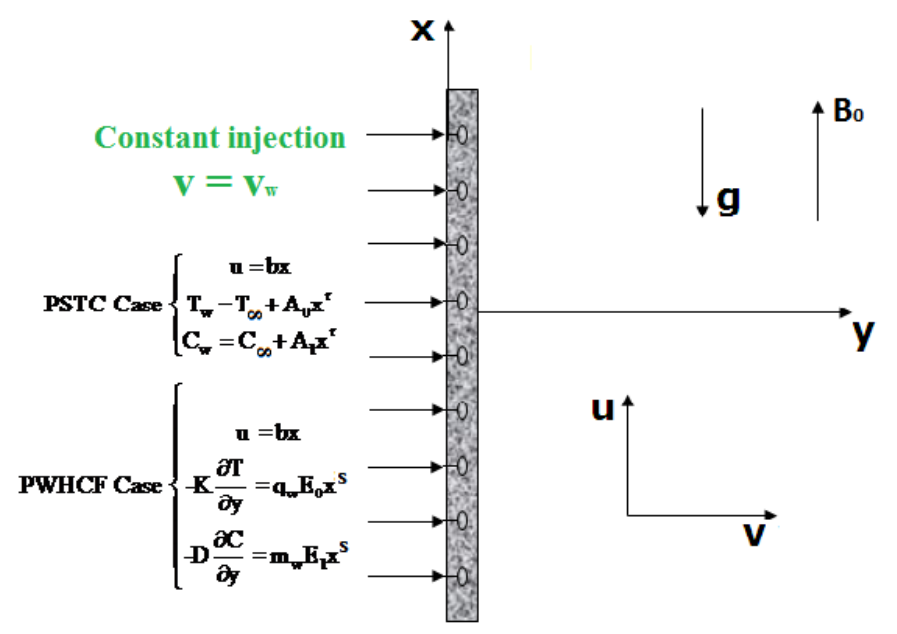

Figure 1. Physical configuration. 
Under all the above assumptions along with Boussinesq approximation, the governing equations are given by:

$\frac{\partial u}{\partial x}+\frac{\partial v}{\partial y}=0$,

$u \frac{\partial u}{\partial x}+v \frac{\partial u}{\partial y}=\frac{1}{\rho} \frac{\partial}{\partial y}\left(\mu \frac{\partial u}{\partial y}\right)+g \beta_{T}\left(T-T_{\infty}\right)-g \beta_{C}\left(C-C_{\infty}\right)-\frac{\sigma_{m} B_{0}^{2} u}{\rho}$,

$u \frac{\partial T}{\partial x}+v \frac{\partial T}{\partial y}=\frac{k}{\rho C_{p}} \frac{\partial^{2} T}{\partial y^{2}}+\frac{B u}{\rho C_{p}}\left(T_{\infty}-T\right)-\frac{\sigma_{m} B_{0}^{2} u^{2}}{\rho c_{p}}+\frac{D_{m} K_{T}}{C_{s} C_{p}} \frac{\partial^{2} C}{\partial y^{2}}$,

$u \frac{\partial C}{\partial x}+v \frac{\partial C}{\partial y}=D_{m} \frac{\partial^{2} C}{\partial y^{2}}+\frac{D_{m} K_{T}}{T_{m}} \frac{\partial^{2} T}{\partial y^{2}}$

and the viscosity parameter $\theta_{r}$ is defined by Seddeek[3],

$\theta_{r}=\frac{T_{r}-T_{\infty}}{T_{w}-T_{\infty}}=-\frac{1}{\gamma\left(T_{w}-T_{\infty}\right)}$.

where the velocity components in the direction of $x$ and $y$ are respectively $u$ and $v, C_{p}$ (is the specific heat at constant pressure), $\rho$ (is the free stream density), $\mu$ (is the fluid viscosity), $g$ (is the acceleration due to gravity), $\mathrm{T}$ (is the temperature inside the boundary layer), $\beta_{\mathrm{T}}$ (is the coefficient of thermal expansion), $\beta_{\mathrm{C}}$ (is the coefficient of expansion with concentration), $C$ (is the concentration of the foreign fluid inside the boundary layer, $k$ (is the thermal conductivity), $D_{m}$ (is the coefficient of mass diffusivity), $T_{m}$ (is the mean fluid temperature), $K_{T}$ (is the thermal diffusion ratio) and $C_{s}$ (is the concentration susceptibility), $B u\left(T_{\infty}-T\right)$ is assumed to be the amount of heat generated or absorbed per unit volume, here $B$ is a constant depends on heat source or sink. $T_{\infty}$ is the ambient temperature, $C_{\infty}$ is the ambient concentration, $B_{0}$ is the uniform transverse magnetic field, $\sigma_{m}$ is the electrical conductivity. $a, T_{r}$ are the constants depends on the thermal property of the fluid $\gamma, T_{w}$ is the accelerating wall temperature.

To analyze the flow characteristics of the physical system for different boundary conditions, we considered in two different manners: (i) PSTC (Prescribed Surface Temperature and Concentration) and (ii) PWHCF (Prescribed Wall Heat and Concentration Flux).

(i) Prescribed Surface Temperature and Concentration (PSTC) Case.

In this case, the temperature and concentration of the boundary surface of the vertical plate at every point are considered as a polynomial of degree ' $r$ ' and the corresponding boundary conditions on velocity, temperature, and concentration profiles are given by:

$u=b x, v=v_{w}, T_{w}=T_{\infty}+A_{0} x^{r}, C_{w}=C_{\infty}+A_{1} x^{r} \quad$ at $y=0$,

$u=0, T=T_{\infty}, C=C_{\infty} \quad$ as $y \rightarrow \infty$,

where ' $b$ ' is the stretching rate of accelerating surface and $A_{0} \& A_{1}$ are choosing constants. The governing equations (1) - (4) of the physical model can solve numerically by introducing the following similarity variable $\eta$ and the non-dimensional variables $f, \theta, H$. (See Acharya et al. [2]).

$\psi=(v b)^{\frac{1}{2}} x f(\eta), \quad \eta=\left(\frac{b}{v}\right)^{\frac{1}{2}} y, \quad \theta(\eta)=\frac{T-T_{\infty}}{T_{w}-T_{\infty}}, \quad H(\eta)=\frac{C-C_{\infty}}{C_{w}-C_{\infty}}$,

where the stream function $\psi(x, y)$ is defined in such way that $u=\frac{\partial \psi}{\partial y}$ and $v=-\frac{\partial \psi}{\partial x}$ and the following are the corresponding components of velocity.

$u=b x f^{\prime}(\eta), v=-(b v)^{\frac{1}{2}} f(\eta)$.

Substituting Eqs. (8) \& (9) in Eqs. (2) - (4), we obtained the transformed nondimensional non-linear coupled ordinary differential equations as follows:

$f^{\prime \prime \prime}-\left(\frac{1}{\theta-\theta_{r}}\right) \theta^{\prime} f^{\prime \prime}-\left(\frac{\theta}{\theta_{r}}-1\right)\left(f f^{\prime \prime}-f^{\prime 2}\right)+M^{2} f^{\prime}\left(\frac{\theta}{\theta_{r}}-1\right)=\left(\frac{\theta}{\theta_{r}}-1\right)($ Gs $\theta-$ Gc $H)$, 
$\theta^{\prime \prime}+\operatorname{Pr} f \theta^{\prime}-(r+\delta) \operatorname{Pr} f^{\prime} \theta=-\operatorname{Pr} D f H^{\prime \prime}+E M^{2} \operatorname{Pr}^{\prime 2}$,

$H^{\prime \prime}+S c f H^{\prime}-r S c f^{\prime} H=-S c S r \theta^{\prime \prime}$,

and the appropriate non-dimensional transformed boundary conditions are:

$f(0)=-\frac{v_{w}}{(b v)^{\frac{1}{2}}}=-m, \quad f^{\prime}(0)=1, \quad \theta=1, \quad H=1 \quad$ at $y=0$,
$f^{\prime}=0, \quad \theta=0, H=0 \quad$ as $y \rightarrow \infty$.

(ii) Prescribed Wall Heat and Concentration Flux (PWHCF) Case.

For this case, the corresponding boundary conditions on velocity, temperature, and concentration profiles are as follows:

$u=b x, \quad v=v_{w}, \quad-k \frac{\partial T}{\partial y}=q_{w} E_{0} x^{s},-D \frac{\partial C}{\partial y}=m_{w} E_{1} x^{s} \quad$ at $y=0$,

$u=0, T=T_{\infty}, C=C_{\infty}$ as $y \rightarrow \infty$,

where $E_{0}, E_{1}$ are choosing constants, ' $s$ ' is the parameter of heat flux.

The similarity variable $\eta$ and the non-dimensional variables $f, g, h$ introduced by Acharya et al. [2] to transform the Eqs. (1) -(4).

$\psi=(v b)^{\frac{1}{2}} x f(\eta), \quad \eta=\left(\frac{b}{v}\right)^{\frac{1}{2}} y, T-T_{\infty}=\frac{E_{0} x^{s}}{k}\left(\frac{v}{a}\right)^{\frac{1}{2}} g(\eta), C-C_{\infty}=\frac{E_{1} x^{s}}{D}\left(\frac{v}{a}\right)^{\frac{1}{2}} h(\eta)$,

Substituting Eqs. (9) \& (17) in Eqs. (2) - (4), we obtained the transformed nondimensional non-linear coupled ordinary differential equations as follows:

$f^{\prime \prime \prime}-\left(\frac{1}{g-g_{r}}\right) g^{\prime} f^{\prime \prime}-\left(\frac{g}{g_{r}}-1\right)\left(f f^{\prime \prime}-f^{\prime 2}\right)+M^{2} f^{\prime}\left(\frac{g}{g_{r}}-1\right)=\left(\frac{g}{g_{r}}-1\right)($ Gs $g-$ Gc $h)$,

$g^{\prime \prime}+\operatorname{Pr} f g^{\prime}-(s+\delta) \operatorname{Pr} f^{\prime} g=-\operatorname{Pr} D f h^{\prime \prime}+E M^{2} \operatorname{Pr} f^{\prime 2}$,

$h^{\prime \prime}+S c f h^{\prime}-s S c f^{\prime} h=-S c S r g^{\prime \prime}$,

The appropriate non-dimensional transformed boundary conditions are:

$f(0)=-\frac{v_{w}}{(b v)^{\frac{1}{2}}}=-m, f^{\prime}(0)=1, g=-1, h=-1 \quad$ at $y=0$,

$f^{\prime}=0, \quad \theta=0, \quad H=0 \quad$ as $y \rightarrow \infty$.

where prime represents differentiation with respect to $\eta$ and the non-dimensional parameters involved in the above equations are defined in nomenclature.

\subsection{Method of solution.}

For the PSTC case, the Eqs. (10) - (12) along with the boundary conditions (13) \& (14) are the functions of $f(\eta), \theta(\eta) \& H(\eta)$ and similarly for the PWHCF case, the Eqs. (18) (20) along with the boundary conditions (21) \& (22) are the functions of $f(\eta), g(\eta) \& h(\eta)$ and which are to be determined. Since the ODE's are highly non-linear, coupled, and involved variable coefficient like transcendental terms as well as the corresponding boundary conditions so that the known methods pertaining to the analytical nature of the solution cannot be applied. To overcome the difficulty raised in the analytical method here, we adopt the numerical method to find the solution by transforming the higher-order coupled non-linear ODE's into a system of first-order ODE's and these first-order ODE's can be solved by Runge-Kutta-Fehlberg explicit method and second-order accuracy of Newton-Raphson method. The combination of these two numerical techniques to estimate an approximate solution to the problem is referred to as the shooting technique. We developed these computations using software like Mat Lab. 
The computational work is carried out for an accuracy of $10^{-6}$, i.e., the difference between the two iterative successive values is less than the prescribed accuracy of $10^{-6}$.

\section{Results and Discussion}

The main aim of the present work is to understand the effect of the external constraint of magnetic field on the characteristics of velocity, temperature, and concentration over an accelerating surface under the influence of temperature-dependent viscosity; the study of thermal diffusion and diffusion thermal convection in both the cases of PSTC and PWHCF. The numerical results of these equations are drawn using Mat Lab software and which are represented graphically in Figures 2-15 to illustrate the variations of physical parameters like Hartmann number, viscosity parameter, Prandtl number, Eckert number, buoyancy parameters (both thermal and mass), Soret and Dufour numbers. In all computations, we employ the fixed values of non-dimensional parameters $\operatorname{Pr}=0.7, E=1, M=0.5, G s=0.1, \quad G c=$ $0.1, \quad \theta_{r}=3.0, \quad g_{r}=3.0, \quad D f=0.5, \quad S r=0.2, \quad S c=0.6, \quad r=1, \quad s=1, \quad \delta=$ $-5.0, m=-0.5$ in entire study except the varied values as displayed in respective figures. The present numerical results are compared with the published data in the absence of a magnetic field, i.e., for $M=0.0$, and found to be in good agreement with the earlier work of Seddeek [7].

\subsection{Effect of viscosity parameter $\left(\theta_{r}\right)$.}

The numerical results for dimensionless velocity, temperature, and concentration for various values viscosity parameter $\theta_{r}$ are graphed in Figures 2-4, respectively. The enhancement of the viscosity parameter $\theta_{r}$ diminishes the flow velocity, and the decrement is more in the case of PSTC compare to that of PWHCF case; this is because of the heat transfer between far away from the moving vertical plate and the wall is decreased or the higher viscosity reduces the fluid flow rate, which is shown in Figure 2. But we can observe the opposite behavior of temperature profile with an increase of the viscosity parameter $\theta_{r}$ in Figure 3. With an increase of temperature, the fluid becomes lighter, and hence the heat transfer is less in the case of PSTC compared to that of PWHCF case. Similarly, the effect of the viscosity parameter $\theta_{r}$ is very low in the case of concentration because of how the viscosity parameter $\theta_{r}$ indirectly affects the concentration through temperature and momentum equations, which is shown in Figure 4.

\subsection{Effect of Prandtl number(Pr).}

The change of the Prandtl number is due to variations of thermal diffusivity or viscosity. The enhancement of fluid viscosity decelerates the flow velocity of the fluid and the temperature of the fluid, illustrated in Figures 5 and 6, respectively. Whereas quite the opposite trend is observed from Figure 7 in the case of concentration profile, i.e., with the higher values of Prandtl number $\mathrm{Pr}$ results thin the reduction of mofluid flow movement, the dimensionless concentration gradually increases.

\subsection{Effect of Hartmann number(M).}

In Figure 8, it is observed that with the enhancement of Hartmann numberMreduces the velocity due to stretching of the fluid and due to the Lorentz force, which resists the flow 
velocity of the fluid. An opposite behavior can be observed in the case of temperature and concentration profiles for variation in Hartmann number from Figures 9 and 10, respectively. The flow temperature increases due to an enhancement of the magnetic field's intensity, and hence it reduces the rate of heat convection and concentration in the fluid flow, for very small values of the Hartmann number i.e. $M=0.0$, our present results are satisfied with the earlier work of Seddeek [7]. Finally, it is concluded that the effect of Hartmann number $M$ is more in the case of PWHCF and compared with that of PSTC case for velocity, temperature, and concentration profiles because of adiabatic and boundary conditions maintained at the accelerating wall.

\subsection{Effect of Soret and Dufour numbers $(\operatorname{Sr} \& D f)$.}

The variation of dimensionless temperature and concentration is depicted in Figures 11 and 12, respectively, for different values of Soret number $\mathrm{Sr}$. The enhancement of Soret number due to the increase in the temperature difference $\Delta T$; while concentration difference kept as constant or decreased in the concentration difference $\Delta C$; while the temperature difference kept as constant. Similarly, the variations of dimensionless velocity and temperature for different values of Dufour number are represented by Figures 13 and 14, respectively. In this case, the increases of Dufour number due to higher values of $\Delta C$ or lower values of $\Delta T$ results increase the flow velocity and temperature of the fluid. Whereas in the case of concentration profile, a reverse trend can be observed from Figure 15. Here, Soret and Dufour numbers' effect is very less on velocity profile for both cases than that of the temperature and concentration profiles. This is due to the momentum equation is not directly affected by Soret and Dufour numbers.

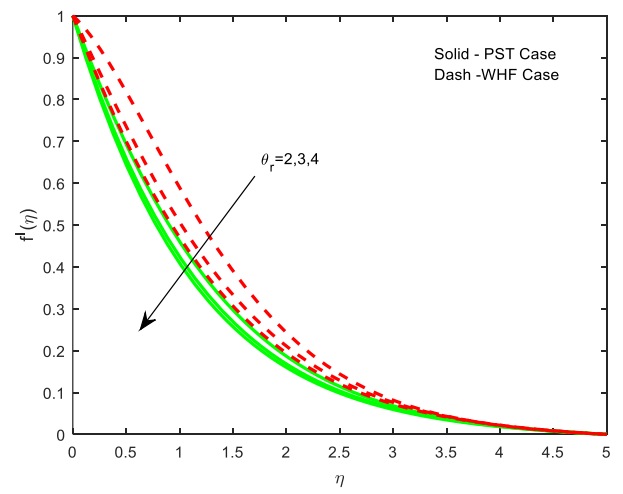

Figure 2. Velocity variations of viscosity parameter.

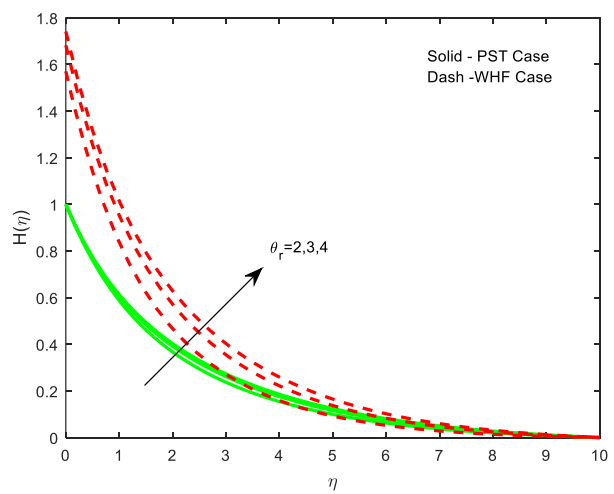

Figure 4. Concentration variations of viscosity parameter.

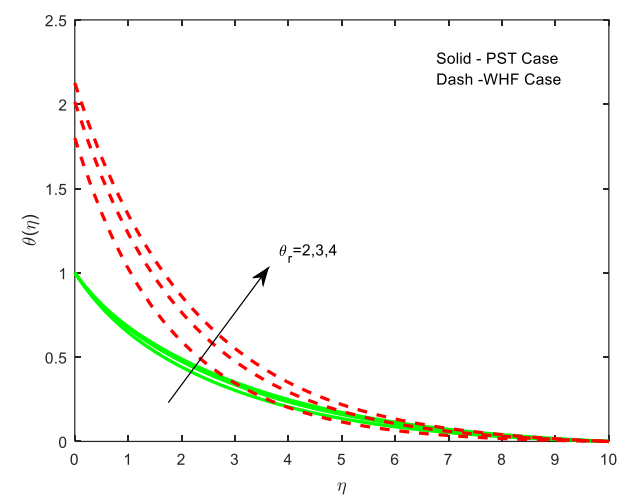

Figure 3. Temperature variations of viscosity parameter.

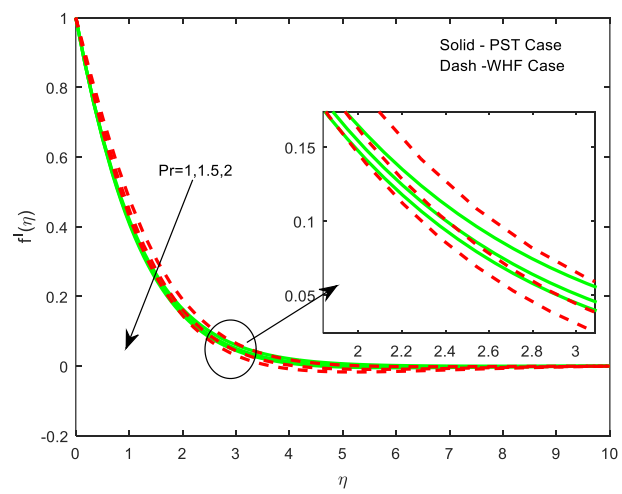

Figure 5. Velocity variations of Prandtl number 


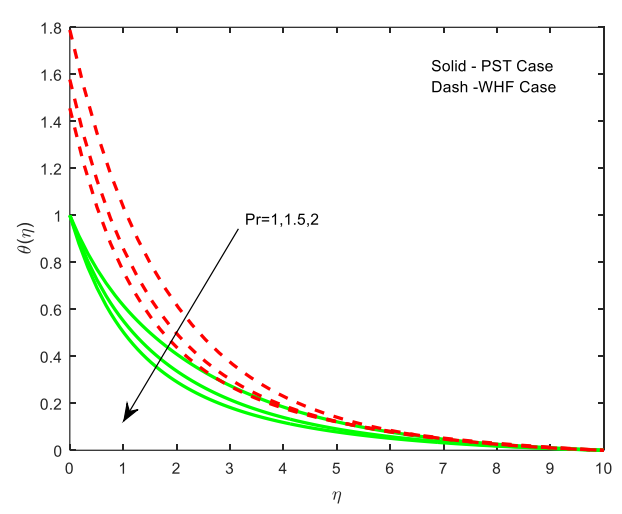

Figure 6. Temperature variations of Prandtl number.

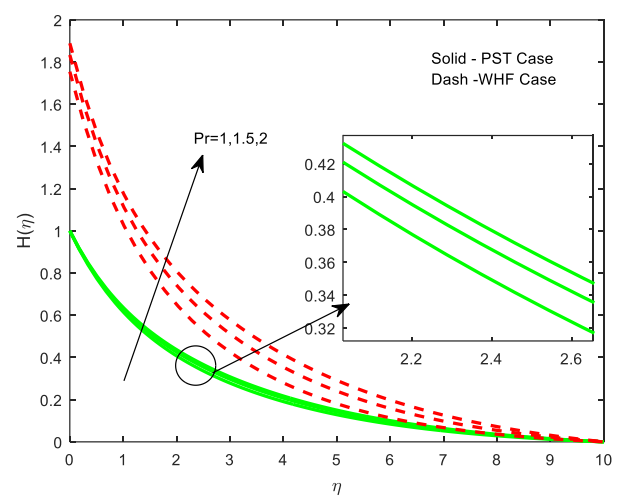

Figure 8. Velocity variations of magnetic number.

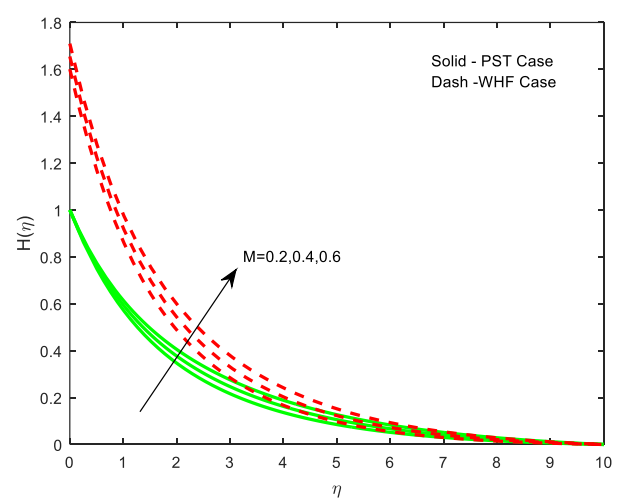

Figure 10. Concentration variations of magnetic number.

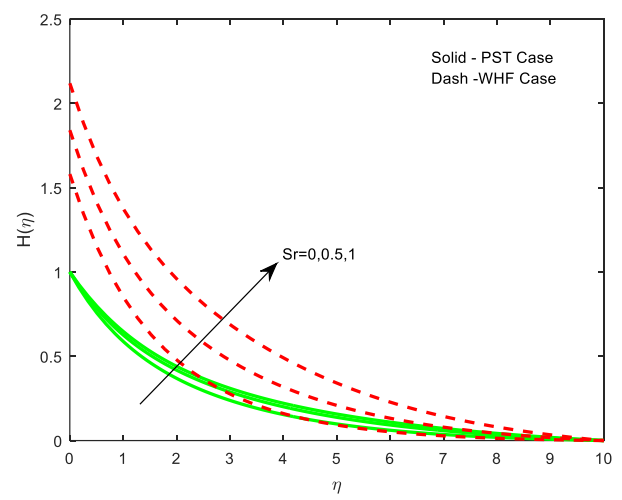

Figure 12. Concentration variations of Soret number.

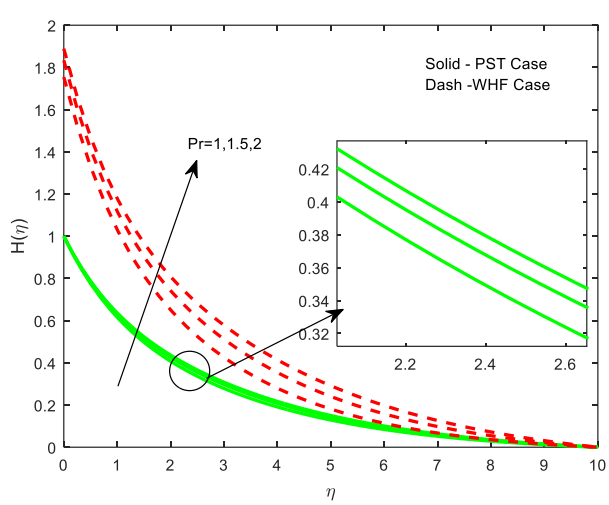

Figure 7. Concentration variations of Prandtl number.

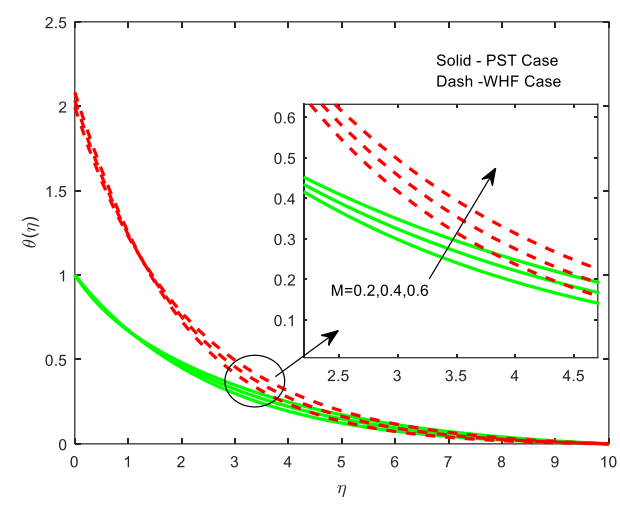

Figure 9. Temperature variations of magnetic number

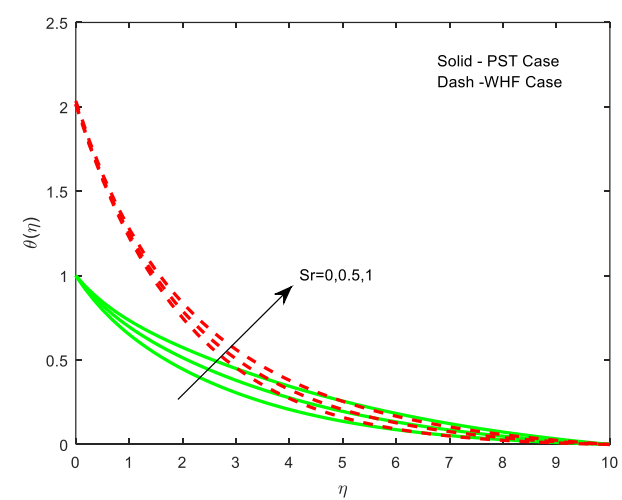

Figure 11. Temperature variations of Soret number.

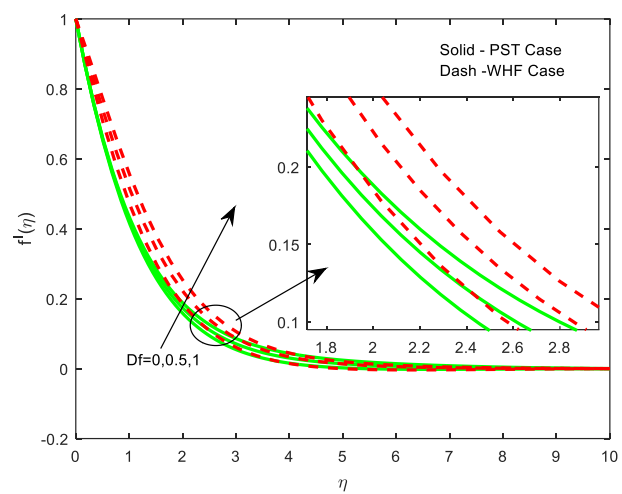

Figure 13. Velocity variations of Dufour number. 


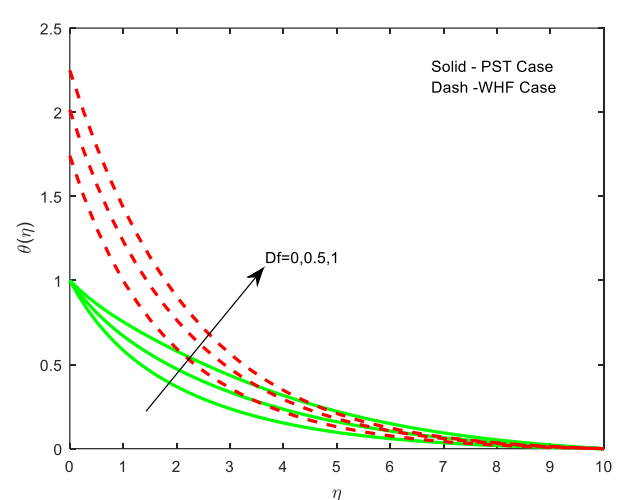

Figure 14. Temperature variations of Dufour number.

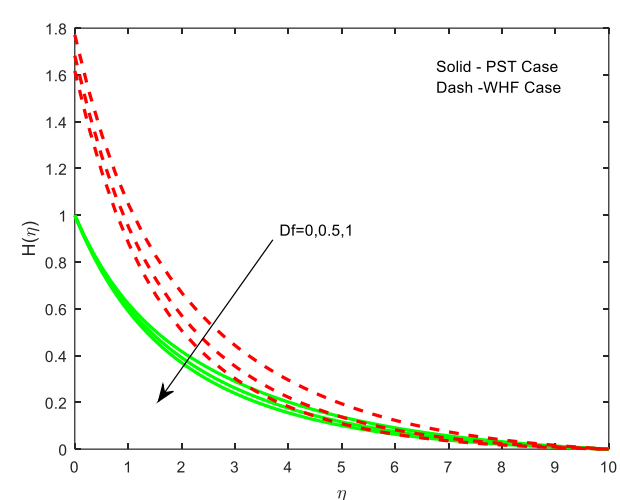

Figure 15. Concentration variations of Dufour number.

\section{Conclusions}

The effects of variable viscosity, Soret and Dufour on double-diffusive (temperature and concentration) mixed convection flow in the presence of MHD over an accelerating surface have been studied numerically. The governing equations are transformed into a set of coupled and highly non-linear ordinary differential equations in the non-dimensional form using the similarity transformation. The shooting technique is used to solve these differential equations numerically. The computed numerical results are presented to analyze the fluid's flow, mass, and heat transfer characteristics under the influence of various physical parameters. The following conclusions can be drawn from the present study: With an increase in the transverse magnetic field's intensity, the solutal and thermal boundary layer increases and opposes the flow velocity; The flow velocity will reduce with an increase in the temperature-dependent viscosity parameter $\left(\theta_{r}\right)$ whereas it enhances the dimensionless temperature and concentration; An increase in Dufour number $(D f)$ results in the enhancement of velocity and temperature gradients whereas it diminishes the concentration profile and with an increase of Soret number $(\mathrm{Sr})$ leads the reduction in temperature and increase in the concentration profile; Higher values of Prandtl number $(P r)$ decrease the velocity and temperature profile, whereas the trend is reversed in the case of concentration profile.

\section{Funding}

This research received no external funding.

\section{Acknowledgments}

The authors are thankful to the research center, Department of Mathematics, M. S. Ramaiah Institute of Technology, Bangalore, affiliated to VTU, for their valuable support to our research work.

\section{Conflicts of Interest}

The authors declare no conflict of interest.

\section{References}

1. Lai, F.C.; Kulacki, F.A. The effect of variable viscosity on convective heat transfer along a vertical surface in a saturated porous medium. Int. J. Heat Mass Transfer 1990, 33, 1028-1031, https://doi.org/10.1016/00179310(90)90084-8. 
2. Acharya, M.; Singh, L.P.; Dash, G.C. Heat and mass transfer over an accelerating surface with heat source in presence of suction and blowing. International Journal of Engineering Science 1999, 37, 189-211, https://doi.org/10.1016/S0020-7225(98)00064-0.

3. Seddeek, M.A. The effect of variable viscosity on hydromagnetic flow and heat transfer past a continuously moving porous boundary with radiation. International Communications in Heat and Mass Transfer 2000, 27, 1037-1046, https://doi.org/10.1016/S0735-1933(00)00183-4.

4. Das, S.; Jana, R.N. Natural convective magneto-nanofluid flow and radiative heat transfer past a moving vertical plate. Alexandria Engineering Journal 2015, 54, 55-64, https://doi.org/10.1016/j.aej.2015.01.001.

5. Eckert, E.R.G.; Drake, R.M., Jr. Analysis of heat and mass transfer; Hemisphere Publishing: United States, 1987.

6. Kafoussias, N.G.; Williams, E.W. Thermal-diffusion and diffusion-thermo effects on mixed free-forced convective and mass transfer boundary layer flow with temperature dependent viscosity. International Journal of Engineering Science 1995, 33, 1369-1384, https://doi.org/10.1016/0020-7225(94)00132-4.

7. Seddeek, M.A. Thermal-diffusion and diffusion-thermo effects on mixed free-forced convective flow and mass transfer over an accelerating surface with a heat source in the presence of suction and blowing in the case of variable viscosity. Acta Mechanica 2004, 172, 83-94, https://doi.org/10.1007/s00707-004-0139-5.

8. Reddy, M.G.; Reddy, N.B. Finite element analysis of soret and dufour effects on unsteady MHD free convection flow past an impulsively started vertical porous plate with viscous dissipation. Journal of Naval Architecture and Marine Engineering 2011, 8, 1-12, https://doi.org/10.3329/jname.v8i1.3507.

9. Girinath Reddy, M.; Dinesh, P.A.; Sandeep, N. Effects of variable viscosity and porosity of fluid, Soret and Dufour mixed double diffusive convective flow over an accelerating surface. IOP Conference Series: Materials Science and Engineering 2017, 263, 062012, https://doi.org/10.1088/1757-899X/263/6/062012.

10. Girinath Reddy, M.; Dinesh, P.A. Double diffusive convection and internal heat generation with Soret and Dufour effects over an accelerating surface with variable viscosity and permeability. Advances in Physics Theories and Applications 2018, 69, 7-25.

11. Ullah, K.S.; Ali, N.; Abbas, Z. Soret and dufour effects on hydromagnetic flow of Eyring-Powell fluid over oscillatory stretching surface with heat generation/absorption and chemical reaction. Thermal Science 2018, 22, 533-543, https://doi.org/10.2298/TSCI150831018U.

12. Jain, S.; Choudhary, R. Soret and Dufour Effects on Thermophoretic MHD Flow and Heat Transfer Over a Non-linear Stretching Sheet with Chemical Reaction. International Journal of Applied and Computational Mathematics 2018, 4, 50, https://doi.org/10.1007/s40819-018-0481-2.

13. Idowu, A.S.; Falodun, B.O. Soret-Dufour effects on MHD heat and mass transfer of Walter's-B viscoelastic fluid over a semi-infinite vertical plate: spectral relaxation analysis. Journal of Taibah University for Science 2019, 13, 49-62, https://doi.org/10.1080/16583655.2018.1523527.

14. Huang, C.-J. Influence of non-Darcy and MHD on free convection of non-Newtonian fluids over a vertical permeable plate in a porous medium with soret/dufour effects and thermal radiation. International Journal of Thermal Sciences 2018, 130, 256-263, https://doi.org/10.1016/j.ijthermalsci.2018.04.019.

15. Babu, M.; Sandeep, D.N. MHD non-Newtonian fluid flow over a slendering stretching sheet in the presence of cross-diffusion effects. Alexandria Engineering Journal 2016, 55, http://dx.doi.org/10.5098/hmt.10.3.

16. Irfan, M.; Khan, M.; Khan, W.A. Impact of non-uniform heat sink/source and convective condition in radiative heat transfer to Oldroyd-B nanofluid: A revised proposed relation. Phys. Lett. A 2019, 383, 376382, https://doi.org/10.1016/j.physleta.2018.10.040.

17. Ahmed, J.; Khan, M.; Ahmad, L. Significance of thermophoresis, thermal-diffusion and diffusion-thermo on the flow of Maxwell liquid film over a horizontal rotating disk. Phys. Scr. 2019, 94, 095003, https://doi.org/10.1088/1402-4896/ab120d.

18. Nalinakshi, N.; Dinesh, P.A. Thermo-Diffusion and Diffusion-Thermo Effects for a Forchheimer Model with MHD Over a Vertical Heated Plate. In Advances in Fluid Dynamics, Springer: 2020; 343-361, https://doi.org/10.1007/978-981-15-4308-1_27.

19. Sravan Kumar, T.; Dinesh, P. A.; Makinde, O. D. Impact of Lorentz force and viscous dissipation on unsteady nanofluid convection flow over an exponentially moving vertical plate. Mathematical Models and Computer Simulations 2020, 12, 631-646, https://doi.org/10.1134/S2070048220040110.

20. Krishna, M.V.; Swarnalathamma, B.V.; Chamkha, A.J. Investigations of Soret, Joule and Hall effects on MHD rotating mixed convective flow past an infinite vertical porous plate. Journal of Ocean Engineering and Science 2019, 4, 263-275, https://doi.org/10.1016/j.joes.2019.05.002.

21. Goud, B.S.; Babu, B.S.; Shekar, M.N.R.; Srinivas, G. Mass Transfer Effects on MHD Flow through Porous Medium past an Exponentially Accelerated Inclined Plate with Variable Temperature and Thermal Radiation. International Journal of Thermofluid Science and Technology 2019, 6, 19060402, https://doi.org/10.36963/IJTST.19060402.

22. Abdal, S.; Ali, B.; Younas, S.; Ali, L.; Mariam, A. Thermo-Diffusion and Multislip Effects on MHD Mixed Convection Unsteady Flow of Micropolar Nanofluid over a Shrinking/Stretching Sheet with Radiation in the Presence of Heat Source. Symmetry 2020, 12, 49., http://doi.org/10.3390/sym12010049.

23. Tijani Yusuf; Fasogbon. F. Hydromagnetic radiative flow past a vertical oscillating plate with chemical reaction in presence of heat source. Nigerian journal of Mathematics and applications 2019, 26, 45-60. 
24. Sreedhar, G.; Bhupal Reddy, B.R. Chemical Reaction Effect on Unsteady MHD Flow Past an Infinite Vertical Porous Plate in the Presence of Heat Absorption. International Journal of Advanced Research in Engineering and Technology 2019, 10, https://doi.org/10.34218/IJARET.10.1.2019.010.

25. Kempannagari, A.K.; Buruju, R.R.; Naramgari, S.; Vangala, S. Effect of Joule heating on MHD nonNewtonian fluid flow past an exponentially stretching curved surface. Heat Transfer 2020, 49, 3575-3592, https://doi.org/10.1002/htj.21789.

26. Ayegbusi, F.D.; Onwubuoya, C.; Falodun, B.O. Unsteady problem of magnetohydrodynamic heat plus mass transfer convective flow over a moveable plate with effects of thermophoresis and thermal radiation. Heat Transfer 2020, 49, 3593-3612, https://doi.org/10.1002/htj.21790.

27. Dinesh, P.A.; Vasudevamurthy, A.S.; Uma, M. Effects of Forchheimer, MHD and Radiation Absorption for Chemically Reacting Unsteady Dusty Viscoelastic Fluid Couette Flow in an Irregular Channel. In Advances in Fluid Dynamics, Springer: 2020; 999-1012, https://doi.org/10.1007/978-981-15-4308-1_77.

28. Basavaraj, M. S.; Girinath Reddy, M.; Aruna, A.S.; Dinesh P.A. A non-linear mixed convective oscillatory flow over a semi-infinite vertical plate through porous medium under uniform magnetic field. Int. Journal of Advanced Research (IJAR) 2020, 8(6), 308-321, https://dx.doi.org/10.21474/IJAR01/11100.

29. Uma, M.; Dinesh, P.A. Combined effects of Forchheimer, Soret and Dufour on MHD mixed convective dusty viscoelastic Couette flow in an irregular channel. Multidiscipline Modeling in Materials and Structures 2020., https://doi.org/10.1108/MMMS-12-2019-0233.

30. Filahi, I.; Bourich, M.; Hasnaoui, M.; Amahmid, A. Analytical and Numerical Study of Soret and Dufour Effects on Thermosolutal Convection in a Horizontal Brinkman Porous Layer with a Stress-Free Upper Boundary. Mathematical Problems in Engineering 2020, 2020, https://doi.org/10.1155/2020/4046570. 